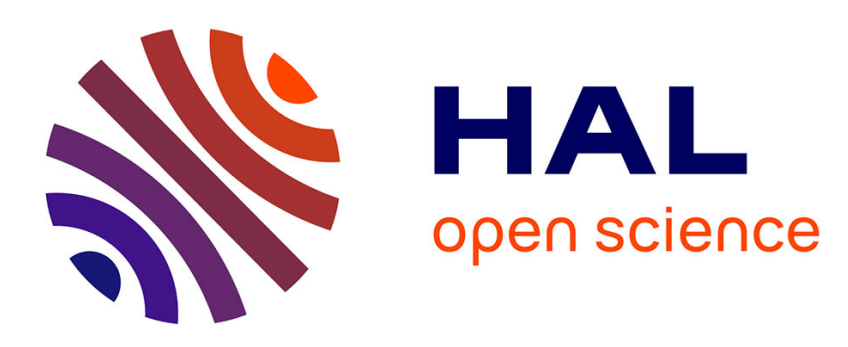

\title{
Identification de paramètres de comportement de membranes en polymères durant le procédé de soufflage
}

Samia Hmida Maamar, Fabrice Schmidt, Laurent Robert, Vincent Velay, Daniel Mercier

\section{- To cite this version:}

Samia Hmida Maamar, Fabrice Schmidt, Laurent Robert, Vincent Velay, Daniel Mercier. Identification de paramètres de comportement de membranes en polymères durant le procédé de soufflage. Matériaux \& Techniques, 2007, 94 (5), pp.371-378. 10.1051/mattech:2007010 . hal-01851430

\author{
HAL Id: hal-01851430 \\ https://hal.science/hal-01851430
}

Submitted on 19 Mar 2019

HAL is a multi-disciplinary open access archive for the deposit and dissemination of scientific research documents, whether they are published or not. The documents may come from teaching and research institutions in France or abroad, or from public or private research centers.
L'archive ouverte pluridisciplinaire HAL, est destinée au dépôt et à la diffusion de documents scientifiques de niveau recherche, publiés ou non, émanant des établissements d'enseignement et de recherche français ou étrangers, des laboratoires publics ou privés. 


\title{
Identification de paramètres de comportement de membranes en polymères durant le procédé de soufflage
}

\author{
S. Hmida-Maamar ${ }^{1}$, F. Schmidt ${ }^{1}$, L. Robert ${ }^{1}$, V. Velay ${ }^{1}$ et D. Mercier ${ }^{2}$ \\ 1 Ecole des Mines d'Albi-Carmaux, Route de Teillet, 81013 Albi CT Cedex 09, France \\ 2 LEHIGH University Mechanical Engineering \& Mechanics Packard Laboratory, 19 Memorial Drive \\ West Lehigh University, Bethlehem PA 18015, USA \\ e-mail : samia.hmida@enstimac.fr
}

Mots-clés :

Soufflage; grande déformation; polymère; stéréo-corrélation; identification; analyse inverse

\section{Key words:}

Bubble inflation large deformation; polymer; stereo-correlation; identification; inverse problem
Résumé - Les propriétés bi-axiales des matériaux tels que les élastomères ou les polymères, utilisés dans des applications mécaniques ou thermoplastiques, sont souvent difficiles à identifier. Pourtant, elles s'avèrent nécessaires pour la simulation des procédés de thermoformage, d'injection-soufflage ou de formage par soufflage. La démarche globale de ce travail de recherche est d'identifier à partir d'extraction de contour et de mesure de champs de surface, et par analyse inverse en utilisant des logiciels de simulations numériques type Eléments finis, le comportement de ce type de matériaux. Le comportement rhéologique et les propriétés mécaniques de tels matériaux peuvent être obtenus en utilisant un rhéomètre permettant le soufflage de bulle. Dans cet article, des résultats expérimentaux obtenus à l'aide d'un rhéomètre élongationnel développé au laboratoire sont présentés. Une description détaillée de la partie expérimentale est faite. Deux méthodes de mesures optiques sont utilisées: (i) une méthode développée à partir d'une mesure de contour avec une seule caméra CCD; (ii) une méthode de mesure de champs par stéréo-corrélation d'images. La modélisation numérique est réalisée en utilisant le logiciel Eléments Finis ABAQUS ${ }^{\circledR}$. Des résultats préliminaires d'identification (prise en compte des conditions aux limites, fonction coût, paramètres du modèle de comportement) sont présentés, ainsi que la validation de ces résultats en réalisant des essais de traction.

\begin{abstract}
Behaviour parameters identification of polymer membranes during bubble inflation process. The biaxial properties of materials such as rubbers or polymers, used in mechanical or thermoplastic applications, are often difficult to identify. However, biaxial properties are useful for the simulation of plastic-processing operations such as blow molding, stretch blow molding or thermoforming. The global approach is to identify the hyperelastic behavior of this type of materials from contour measurements and full field measurements by inverse analysis using numerical simulations such as Finite Element Method. The rheological behavior and the mechanical properties of rubbers and polymers can be obtained by using a bubble inflation rheometer. In this paper, experimental results obtained using elongational rheometer developed in the laboratory are presented. A detailed description of the experimental part is made. Two optical measurement methods are used: (i) measure with only one CCD camera; (ii) the full field technique of images stereocorrelation (2 CCD cameras). Numerical Modeling are realized by using Finite Element software ABAQUS ${ }^{\circledR}$. Preliminary results of the identification (taken into account of the boundary conditions, function cost, parameters of the model of behavior) and validation of these results by carrying out tensile tests are presented.
\end{abstract}

fin de déterminer le comportement rhéologique et les propriétés mécaniques des matériaux tels que les élastomères ou les polymères, utilisés dans des applications mécaniques ou thermoplastiques [1], un rhéomètre élongationnel permettant le soufflage de bulle a été développé [2].

La démarche globale de ce travail de recherche est d'identifier le comportement de ce type de matériaux, à partir du contour de la bulle et de mesure de champs de déformation à la surface de la bulle, et par analyse inverse en utilisant des logiciels de simulations numériques type Eléments Finis. 
Des travaux ont déjà été faits en identification de paramètres de comportement d'élastomère ou de polymère : par exemple, Karama et al. [3] ont étudié le comportement des ballons pressurisées stratosphériques en polymère. Ils ont fait l'identification en grande déformation de la loi de comportement mais seulement à partir de mesure locale de la flèche et des mesures globales de pression. Concernant les mesures de champs, on peut citer les travaux de Marco et al. [4, 5] sur 1 'étude du comportement du PET à partir d'essais de traction biaxiaux. Des mesures de champs par corrélation d'images bidimensionnelles sont faites. En revanche, ces mesures trop bruitées ne pouvaient être utilisées pour identifier les paramètres de la loi de comportement.

Giton et al. [6] ont proposé récemment une étude sur la déchirure d'élastomère et le comportement en traction par mesure de corrélation 2D et identification de paramètres en utilisant une loi hyperélastique type Mooney-Rivlin à trois paramètres. Ils ont identifié les paramètres de cette loi en utilisant deux fonctions coûts associées à deux types de problème : la première est basée sur l'optimisation de la charge appliquée à la cellule, la deuxième en associant l'optimisation précédente à la minimisation du déplacement en surface aux nœuds. Ils ont trouvé une variation importante entre les deux séries de paramètres. Même si la dernière approche assure des résultats réalistes et fiables dans la zone de concentration de contrainte, un travail additionnel pour séparer les deux techniques d'optimisation leur parait nécessaire.

Dans cet article, une description détaillée de la partie expérimentale est réalisée. Des résultats expérimentaux obtenus à l'aide du rhéomètre élongationnel sont présentés. Deux méthodes de mesures optiques sont utilisées : (i) une méthode développée à partir de mesure de contour avec une seule caméra CCD; (ii) une technique de mesure de champs par stéréo-corrélation. La modélisation est réalisée en utilisant le logiciel Eléments Finis ABAQUS ${ }^{\circledR}$. Des résultats préliminaires d'identification sont présentés ainsi que la validation de ces résultats en réalisant des essais de traction sur éprouvette plate.

\section{Équipement expérimental}

Un rhéomètre élongationnel a été développé au sein de notre laboratoire. Il permet de

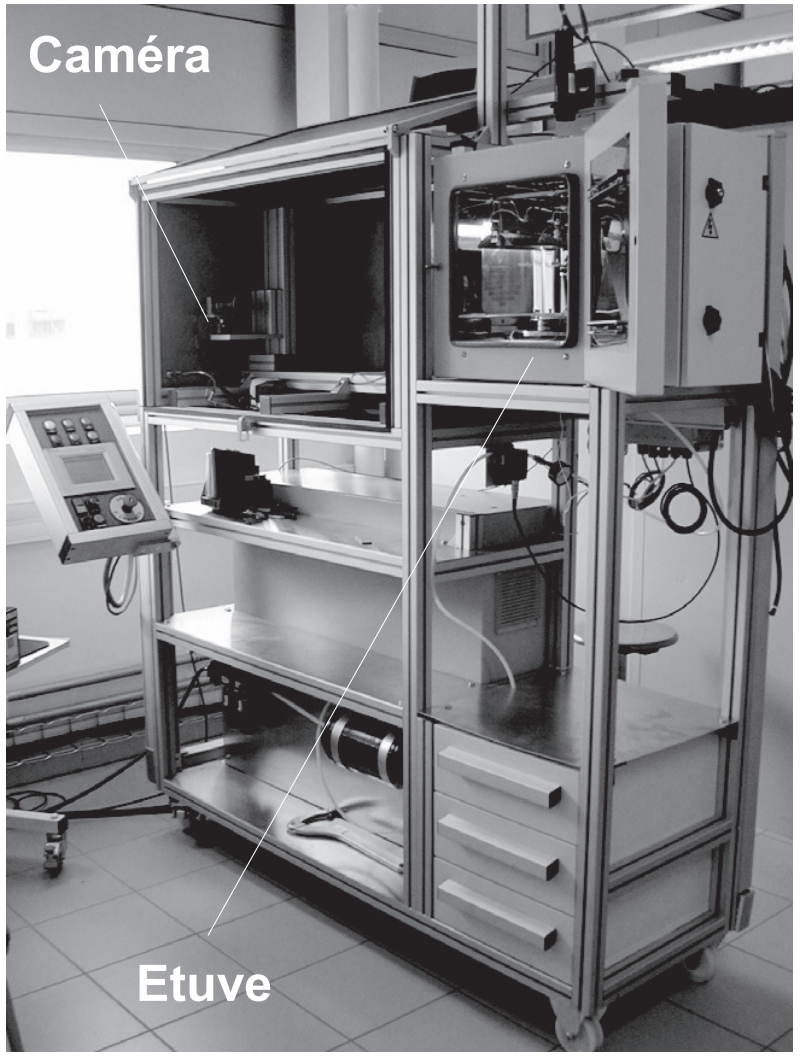

Fig. 1. Rhéomètre élongationnel.

Fig. 1. Elongational rheometer.

gonfler une membrane circulaire en élastomère ou en polymère, maintenue sur son pourtour, en appliquant une pression d'air sur sa face inférieure. Dans le cas de membrane en polymère, une étape de chauffage jusqu'à la température de formage est nécessaire avant d'appliquer la pression d'air.

Le rhéomètre, présenté figure 1 , est constitué : d'une étuve à convection forcée, d'un pyromètre infrarouge pour la mesure de la température au sein de la membrane placé en dessus de l'étuve, d'un capteur de pression pour la mesure de la pression sous la membrane, en dessous de l'étuve et d'un hublot sur le côté de l'étuve pour suivre le procédé de gonflage de membrane.

Afin d'avoir une température homogène au sein de toute la membrane et une bulle bien symétrique au cours du gonflage, trois types de chauffage ont été développés dans l'étuve : pour la régulation thermique, un chauffage convectif et un chauffage conductif à l'aide d'un collier chauffant pour s'assurer de l'homogénéisation de la température au bord de la membrane sont utilisés, et un chauffage radiatif à l'aide de lampes infrarouges pour accélérer le chauffage.

Ce rhéomètre élongationnel est piloté par un ordinateur qui permet d'enregistrer les 

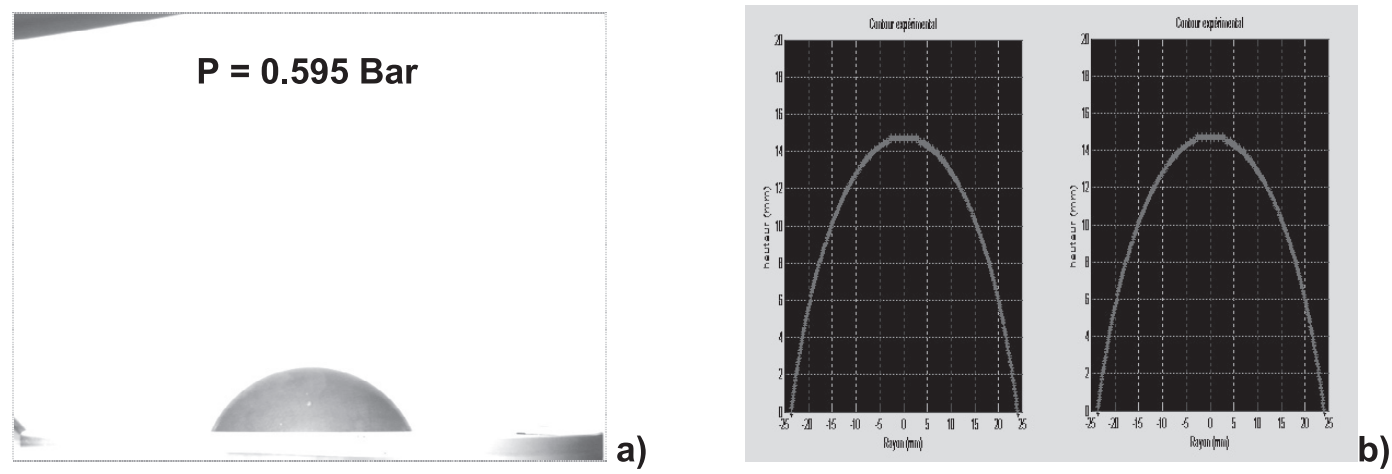

Fig. 2. a) Image de la bulle à une pression $P=0.595$ bar, b) Graphe du contour extrait associé.

Fig. 2. a) Buble's picture at $P=0.595$ bar b) graph of the associated contour.

images au cours du procédé de gonflage, ainsi que la valeur de la pression et de la température pour chaque image. Pour tous les essais, des membranes circulaires en caoutchouc naturel de diamètre $90 \mathrm{~mm}$ et d'épaisseur $1,5 \mathrm{~mm}$ sont utilisées. La membrane étant fixée au bord par un serre-flanc, le diamètre soufflé est de $50 \mathrm{~mm}$.

\section{Méthodes de mesures optiques}

Deux méthodes de mesures optiques ont été utilisées :

- une méthode de mesure avec une seule caméra CCD,

- une technique de mesure de champs par stéréo-corrélation d'images.

\section{Mesure avec une caméra CCD}

Concernant cette méthode de mesure, une seule caméra CCD est utilisée pour l'acquisition des images durant le procédé de gonflage de la membrane. Cette caméra est positionnée parallèlement au plan de symétrie de la bulle. L'image 2D de la bulle est obtenue par ombroscopie (voir Fig. 2a). L'extraction des contours expérimentaux est réalisée par analyse d'images en tenant compte du facteur d'échelle. Ce contour est utilisé dans l'étape d'identification du comportement du matériau détaillée par la suite. La figure $2 b$ présente le contour expérimental dans un repère $(x, y)$ qui correspond à l'image de la bulle de la figure $2 \mathrm{a}$, pour une pression sous la bulle de 0,595 bar. Un algorithme sous Matlab ${ }^{\circledR}$ est utilisé pour l'extraction de contour et le facteur d'échelle vaut dans ce cas $0,19 \mathrm{~mm} /$ pixel.

\section{Mesure de champs par stéréo-corrélation}

La stéréo-corrélation d'images utilise le principe de la vision stéréoscopique, qui permet d'observer la troisième dimension, dont l'appariement entre les images est fait par corrélation de pixels [7]. Elle permet la mesure de la forme $3 \mathrm{D}$ et l'accès au champ de déplacement d'un objet de forme quelconque. Cette technique de mesure de champs est appliquée afin d'obtenir la description tridimensionnelle du champs de déformation de surface [8].

$\mathrm{Au}$ cours de ces essais, deux caméras synchronisées permettent l'acquisition des images durant le procédé de gonflage. Le logiciel Vic-3D ${ }^{\circledR 1}$ est utilisé pour la corrélation des images et l'analyse des résultats. Plusieurs problèmes relatifs aux grandes déformations ont été rencontrés : réflexion spéculaire sur les images qui limite la zone de recouvrement au niveau de la corrélation des paires stéréoscopiques, dégénérescence du mouchetis, etc. Cependant, des résultats préliminaires du champ de déplacement sont obtenus. La figure $3 a$ représente le champ de déplacement suivant la verticale. La figure $3 \mathrm{~b}$ illustre l'évolution de la déformation principale maximale en fonction de la déformation principale minimale pour quatre zones différentes matérialisées par les cercles blancs figure $3 a$. Plusieurs remarques peuvent être formulées : au niveau du pôle, les deux déformations maximale et minimale sont identiques, le comportement est purement équi-biaxial. En revanche, en se rapprochant du bord, la déformation maximale est deux fois plus grande que la déformation minimale, le comportement n'est

\footnotetext{
${ }^{1}$ Vic-3D ${ }^{\odot}$, Correlated Solutions, Inc, http ://www. correlatedsolutions. com
} 

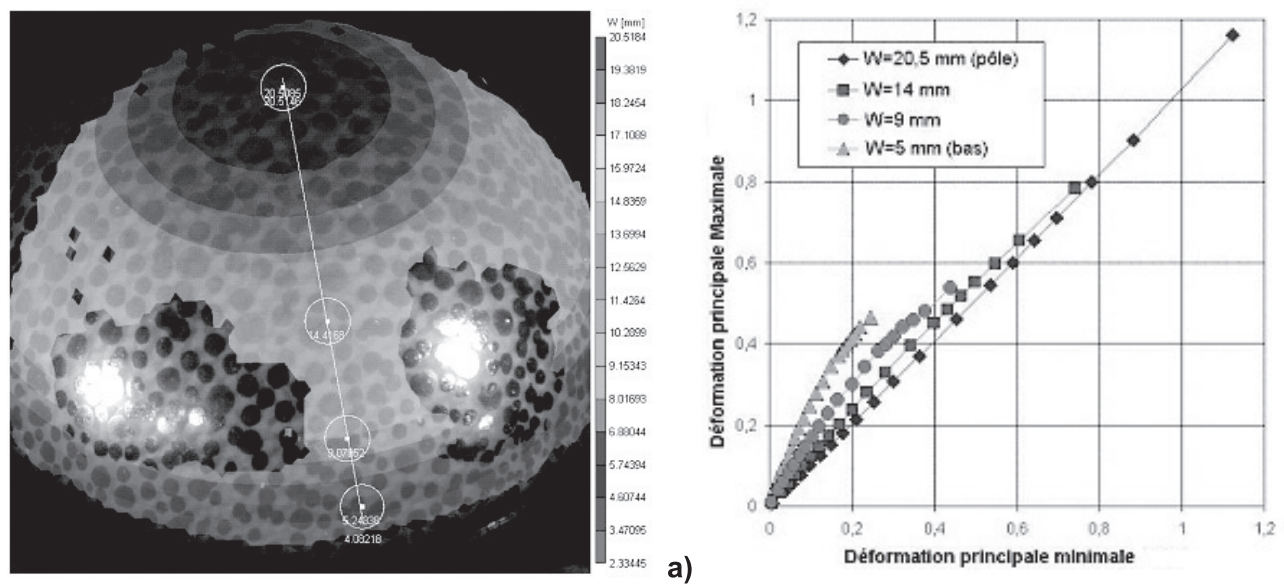

b)

Fig. 3. a) Champ de déplacement suivant la verticale, b) Comportement du matériau au cours du procédé de gonflage.

Fig. 3. a) Displacement vertically b) Material behaviour during blow molding.

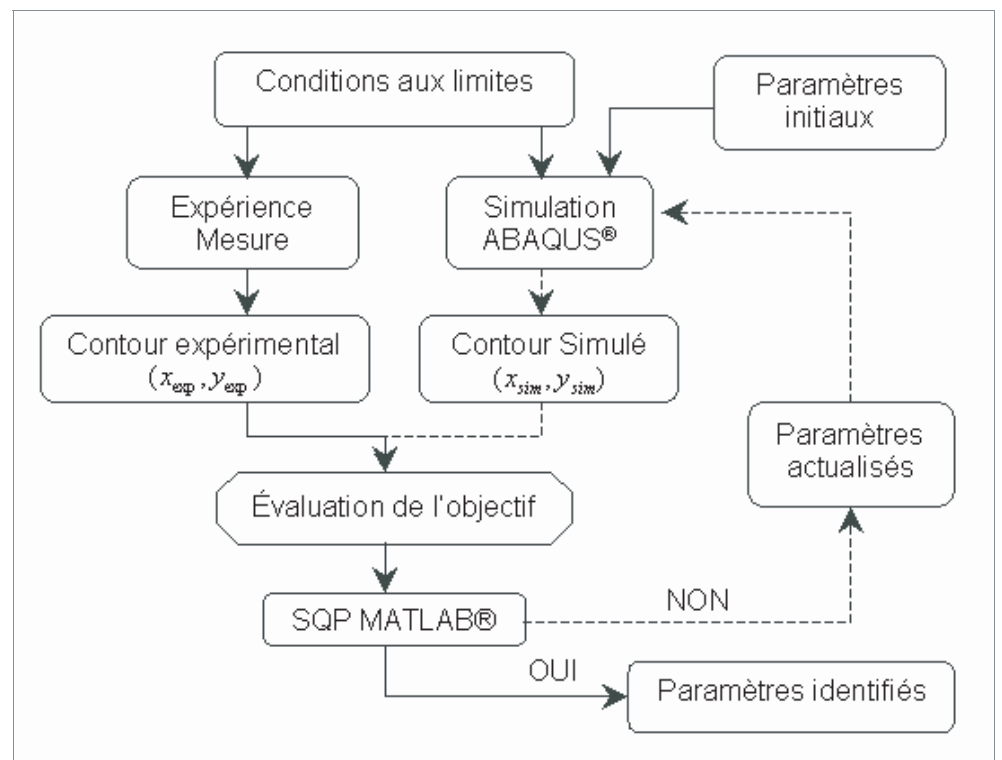

Fig. 4. Algorithme du problème d'identification sur contour.

Fig. 4. Algorithm of contour identification.

plus équi-biaxial, il n'est pas uniforme sur toute la zone de la bulle. Des effets de bord au cours de l'expérience peuvent expliquer ce phénomène qui peut être induit par l'effet du serre-flanc sur la membrane pour des grandes valeurs de pression.

Pour la suite, seuls les résultats issus de la méthode de mesure avec une seule caméra seront utilisés dans le processus d'identification.

\section{Procédure d'identification sur contour}

\section{Principe de l'identification}

L'identification des paramètres du comportement du matériau se base sur une analyse inverse en utilisant le logiciels Eléments Finis $A B A Q U S{ }^{\circledR}$.

Le problème d'identification consiste à résoudre un problème d'optimisation où la fonction à minimiser est une fonction coût qui exprime l'écart entre les données expérimentales et le résultat de la simulation numérique [9]. La figure 4 représente l'algorithme du processus d'identification.

\section{Modélisation et choix de la loi de comportement}

Compte tenu des symétries de la géométrie, des éléments 2D à interpolation quadratiques axisymétriques sont utilisés. Les 


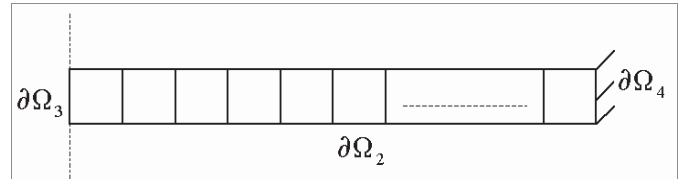

Fig. 5. Modélisation du cas expérimental.

Fig. 5. Modeling of experimental case.

conditions aux limites sont présentées figure 5 :

- axisymétrie au niveau de $\partial \Omega_{3}$,

- encastrement des nœuds sur $\partial \Omega_{4}$,

- application de la pression sur les faces inférieures des éléments de $\partial \Omega_{2}$.

Le maillage comporte un seul élément dans l'épaisseur et 20 éléments sur le rayon.

Une loi de comportement hyperélastique de type Mooney-Rivlin, en général bien représentative du comportement des élastomères et souvent utilisée dans la littérature [10], a été considérée.

De plus, cette loi offre l'avantage de n'avoir que deux paramètres à identifier, ce qui réduit le nombre d'itération du protocole d'identification avant convergence (voir plus loin).

La fonction d'énergie de déformation a l'expression suivante :

$$
w=C_{10}\left(I_{1}-3\right)+C_{01}\left(I_{2}-3\right)
$$

avec : $C_{10}$ et $C_{01}$ les constantes matérielles et $I_{1}$ et $I_{2}$ les invariants du tenseur de Cauchy-Green gauche.

Un résultat d'une simulation directe pour une pression sous membrane $P=$ 0,27 bar est présenté figure 6 , un déplacement axial de $27 \mathrm{~mm}$ est obtenu.

\section{Principe d'optimisation}

Le processus d'identification est mis en place à l'aide du logiciel MATLAB ${ }^{\circledR}$. Il permet de lancer la simulation Eléments Finis (ABAQUS ${ }^{\circledR}$ ), d'extraire les données nécessaires, de définir et d'évaluer la fonction coût. L'optimisation utilise l'algorithme SQP (Programmation Quadratique Séquentielle) déjà implanté dans MATLAB ${ }^{\circledR}$.

Cette méthode itérative est basée sur l'algorithme de Newton/Wilson ; à chaque itération, on est ramené à résoudre un sous problème quadratique jusqu'à ce que les critères de convergence soient atteints [11].
La fonction coût à minimiser est de la forme suivante :

$$
\begin{gathered}
\Phi=\sqrt{\sum_{i=1}^{N}\left(y_{\text {sim }}^{i}-f\left(x_{\text {sim }}^{i}\right)\right)^{2}} \\
\sqrt{\sqrt{\sum_{i=1}^{N}\left(f\left(x_{\text {sim }}^{i}\right)\right)^{2}}}
\end{gathered}
$$

où :

- $\left(x_{\text {sim }}, y_{\text {sim }}\right)$ est le contour simulé,

- $f$ est la fonction d'interpolation polynomiale du contour expérimental $\left(x_{\exp }, y_{\exp }\right)$,

- $N$ est le nombre de points du contour.

\section{Résultats préliminaires}

Pour cinq images acquises à différentes valeurs de pression, différents jeux de paramètres de la loi de comportement sont identifiés.

Les valeurs sont données dans le tableau I pour chaque niveau de pression.

La figure 7 montre la bonne correspondance entre les valeurs expérimentales et calculées.

Il y a une très légère variation des paramètres d'une pression à une autre. Cette différence peut être attribuée à :

- la qualité des images au cours de l'expérience qui peut influer sur l'extraction de contour,

- l'incertitude sur la mesure de pression,

- le choix de la loi de comportement.

Des travaux sont en cours pour résoudre ces différents problèmes ainsi que le calcul de sensibilité par rapport aux paramètres.

\section{Identification sur un essai de traction simple}

Dans le but de valider les résultats précédents d'identification, des essais de traction ont été réalisés sur des éprouvettes rectangulaires

conçues avec le même matériau que les membranes circulaires. Au cours de ces essais, la mesure de champs de déplacement et de déformation est faite à l'aide de la technique de stéréo-corrélation en utilisant le logiciel Vic-3D ${ }^{\odot}$ (voir paragraphe 2.2).

La figure 8 présente la courbe de comportement expérimental et la courbe identifiée après optimisation. L'identification des 


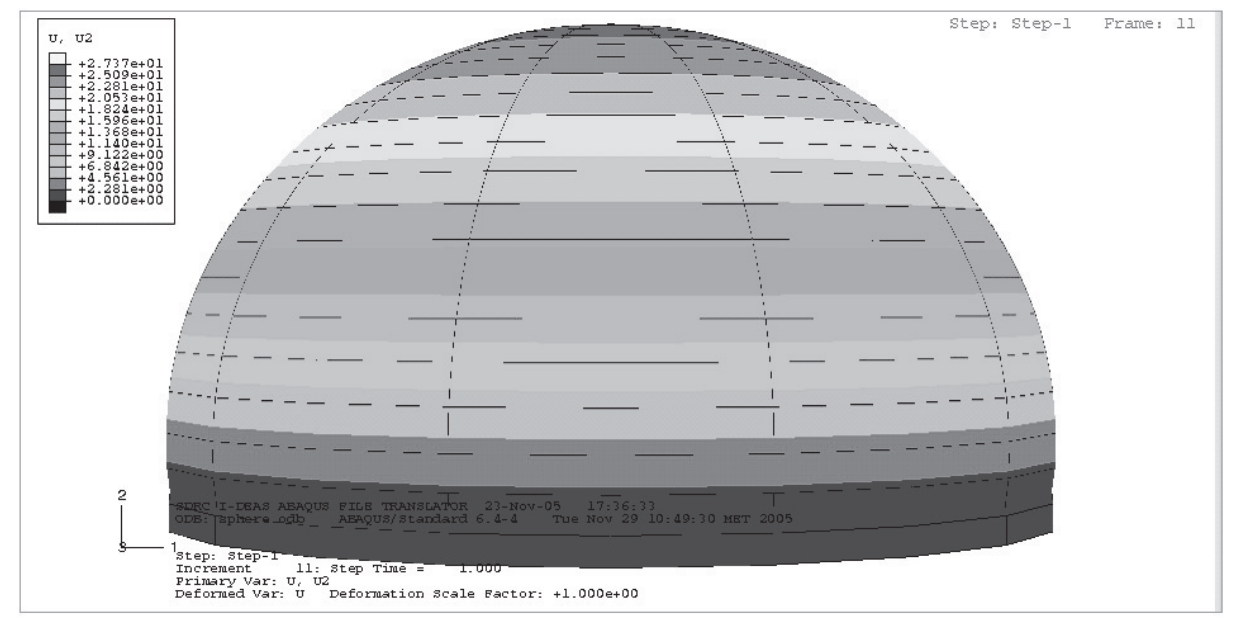

Fig. 6. Simulation numérique avec ABAQUS ${ }^{\circledR}$.

Fig. 6. Numerical modeling by using ABAQUS (registered).

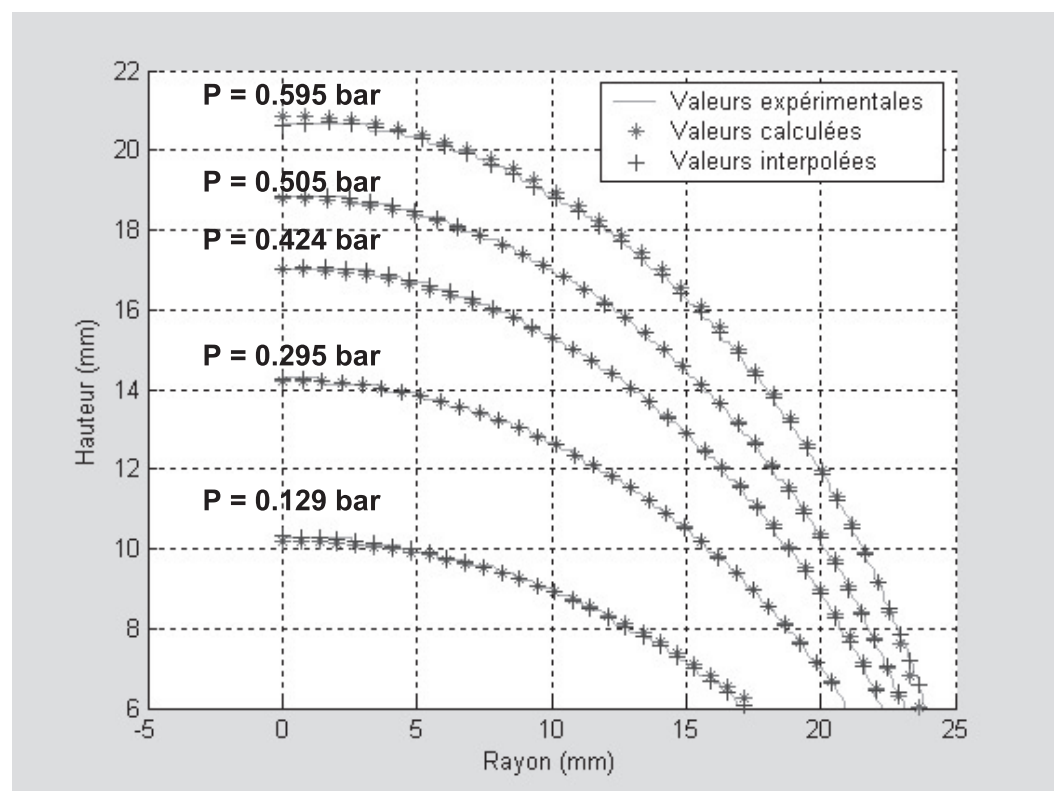

Fig. 7. Comparaison du contour expérimental et calculé.

Fig. 7. Comparison of experimental and calculated contour.

Tableau 1. Identification des paramètres de la loi de comportement. Table 1. Parameters of the behaviour's law identification.

\begin{tabular}{|l|l|l|l|l|l|}
\hline Pression (bar) & 0,129 & 0,295 & 0,424 & 0,505 & 0,595 \\
\hline$C_{10}(\mathrm{MPa})$ & 0,1343 & 0,13677 & 0,13291 & 0,13751 & 0,134 \\
\hline $\mathrm{C}_{01}(\mathrm{MPa})$ & 0,09913 & 0,09651 & 0,09971 & 0,0968 & 0,09954 \\
\hline
\end{tabular}

paramètres de la loi de comportement du matériau, se base sur l'utilisation de l'algorithme SQP afin d'optimiser la fonction coût qui s'exprime comme l'écart entre les deux valeurs de la contrainte : valeur expérimentale connaissant l'effort au cours de l'essai et valeur calculée en fonction des deux para- mètres de la loi de comportement [12]

$$
\sigma=\frac{F}{S_{0}}=2 \cdot\left(\lambda-\frac{1}{\lambda^{2}}\right) \cdot\left(\frac{\partial w}{\partial I_{1}}+\frac{1}{\lambda} \cdot \frac{\partial w}{\partial I_{2}}\right)
$$

où :

- F est l'effort mesuré au cours de l'essai,

- $S_{0}$ est la surface initiale de l'éprouvette,

- $\lambda$ est le taux d'élongation calculé à partir de la valeur de la déformation expérimentale,

- $w$ est la fonction d'énergie de déformation type Mooney-Rivlin utilisée précédemment. 


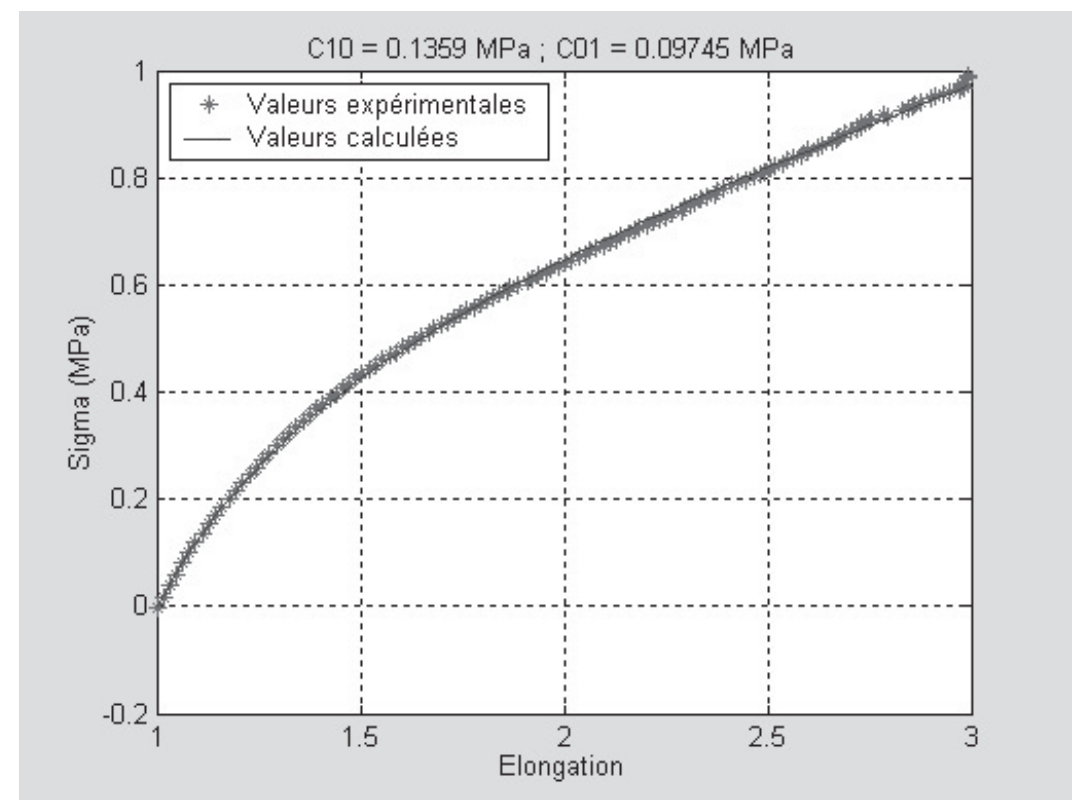

Fig. 8. Identification sur un essai de traction simple.

Fig. 8. Simple traction try identification.

Les paramètres de la loi de comportement identifiés au cours des essais de traction (unidimensionnels) sont $C_{10}=0,1359 \mathrm{MPa}$ et $C_{01}=0,09745 \mathrm{MPa}$. Ces valeurs sont très proches de celles identifiées lors des essais de gonflage bidimensionnels à partir des images et de l'extraction des contours.

\section{Conclusions et perspectives}

Dans cet article, les premiers résultats $d^{\prime}$ identification du comportement d'une membrane en caoutchouc au cours du procédé de gonflage ont été présentés à partir de mesures de contour 2D et par analyse inverse. Une validation des résultats d'identification a été présentée en réalisant des essais de traction simple sur des éprouvettes plates et pour un matériau identique aux membranes circulaires. Des résultats préliminaires de mesures de champ 3D utilisant la stéréo-corrélation ont été obtenus.

Ces mesures offrent des perspectives très intéressantes et novatrices à ce travail. En effet, le comportement de membrane en caoutchouc pourrait être analysé à partir de mesures de champ 3D, et mesures de champ $2 \mathrm{D}$ en réalisant des essais de tractions sur éprouvette plate. Ces travaux permettraient de valider les identifications réalisées par extraction de contour compte tenu du caractère local des informations fournies par les mesures de champs. Cette démarche globale pourrait ensuite être utilisée pour iden- tifier le comportement de membranes en polymère, ce qui nécessiterait 1 'ajout du chauffage au cours des essais et la prise en compte de 1 'effet de la vitesse de déformation dans la loi de comportement.

\section{Remerciements}

Les auteurs tiennent à remercier l'équipe projet constitué de : Jean-Paul Arcens, Jean-Michel Mouys, Didier Ade et Sabine Leroux pour leur collaboration dans ce travail.

\section{Références}

[1] E. Verron, Contribution Expérimentale et Numérique aux Procédés de Moulage par Soufflage et de Thermoformage. Thèse de Doctorat, École Centrale de Nantes, 1997

[2] N. Reuge, F.-M. Schmidt, Y. Lemaoult, M. Rachik, F. Abbe, Polymer Engineering and Science 41 (2001)

[3] P. Vialettes, J.-M. Siguier, P. Guigue, S. Mistou, O. Dalverny, M. Karama, F. Petijean, Mesure par stéréo-corrélation des champs de déplacements et de déformations de ballons stratosphériques pressurisés. Proceedings de Photomécanique'04, Albi, France (2004) 137

[4] L. Chevalier, Y. Marco, Rhéologie 6 (2004) 45

[5] Y. Marco, Caractérisation multi-axiale du comportement et de la microstructure d'un semi-cristallin : Application au cas du PET. Thèse de doctorat, École Normale Supérieure de Cachan (2003) 
[6] M. Giton, A.-S. Bretelle, P. Ienny, Hyperelastic behavior identification by a forward problem resolution : application to a tear test of a silicone-rubber, Proceedings of $8^{\text {th }}$ European Mechanics of Materials Conference, Cachan, France (2005) 193

[7] D. Garcia, Mesure de formes et de champs de déplacements tridimensionnels par stéréocorrélation d'images, Thèse de doctorat, Institut National Polytechnique de Toulouse (2001)

[8] J.-J. Orteu, Mesure 3D de formes et de déformations par stéréovision. Technique de l'ingénieur, traité Génie mécanique, BM 7015 (2002)

[9] D. Lecompte, A. Smits, H. Sol, J. Vantomme, D. Van Hemelrijck, Elastic orthotropic parameter identification by inverse modeling of biaxial tests using Digital Image Correlation, Proceedings of 8th European Mechanics of Materials Conference, Cachan, France (2005) 53

[10] H.-C. Chung, J. Liang, S. Kushiyama, M. Shinozuka, International Journal of NonLinear Mechanics 39 (2004) 691

[11] E. Mathey, Optimisation numérique du refroidissement des moules d'injection de thermoplastiques basée sur la simulation des transferts thermiques par la méthode des éléments frontières, Thèse de doctorat, Université Toulouse III (2004)

[12] G. Marckmann, Contribution à l'étude des élastomères et des membranes soufflées, Thèse de doctorat, École Centrale de Nantes et Université de Nantes (2004) 